\title{
A triple-biomarker approach for the detection of delayed graft function after kidney transplantation using serum creatinine, cystatin C, and malondialdehyde
}

\author{
Isabel Fonseca ${ }^{\mathrm{a}, \mathrm{b}, \mathrm{c}, *}$, Henrique Reguengo ${ }^{\mathrm{d}}$, José Carlos Oliveira ${ }^{\mathrm{d}}$, La Salete Martins ${ }^{\mathrm{a}, \mathrm{b}}$, Jorge Malheiro ${ }^{\mathrm{a}, \mathrm{b}}$, \\ Manuela Almeida ${ }^{\mathrm{a}, \mathrm{b}}$, Josefina Santos ${ }^{\mathrm{a}, \mathrm{b}}$, Leonídio Dias ${ }^{\mathrm{a}}$, Sofia Pedroso a , Luísa Lobato a,b, \\ António Castro Henriques ${ }^{\mathrm{a}, \mathrm{b}}$, Denisa Mendonça ${ }^{\mathrm{c}, \mathrm{e}}$ \\ a Department of Nephrology and Kidney Transplantation, Centro Hospitalar do Porto, Hospital de Santo António, Porto, Portugal \\ ${ }^{\mathrm{b}}$ Unit for Multidisciplinary Investigation in Biomedicine (UMIB), Porto, Portugal \\ ${ }^{c}$ EPIUnit - Institute of Public Health, University of Porto, Porto, Portugal \\ d Department of Clinical Chemistry, Centro Hospitalar do Porto, Hospital de Santo António, Porto, Portugal \\ e Department of Population Studies, Institute of Biomedical Sciences Abel Salazar (ICBAS), University of Porto, Porto, Portugal
}

\section{A R T I C L E I N F O}

\section{Article history:}

Received 2 February 2015

Received in revised form 1 July 2015

Accepted 6 July 2015

Available online $\mathrm{xxxx}$

\section{Keywords:}

Biomarkers

Malondialdehyde

Cystatin C

Delayed graft function

Kidney transplantation

\section{A B S T R A C T}

Introduction: Serum creatinine ( $\mathrm{SCr}$ ) alone does not allow for the early diagnosis of delayed graft function (DGF) following kidney transplantation (KTx).

Objective, design and methods: The diagnostic utility of urinary neutrophil gelatinase-associated lipocalin (uNGAL), serum leptin, malondialdehyde (MD.A), and cystatin C (CysC) for the early detection of DGF was previously evaluated by our group in a prospective cohort study of 40 consecutive adults undergoing KTx. Because no single biomarker achieved adequate sensitivity or specificity for practical purposes, this study was designed to evaluate the combined use of new markers with SCr. Urine and blood samples were collected 8-to-12 h after KTx (day-1). Logistic regression was used to combine the biomarkers, and receiver operating characteristic curves and areas under the curve (AUC-ROC) were generated.

Results: Eighteen recipients developed DGF (dialysis requirement during the first post-transplant week). On day-1, the AUC for SCr to predict DGF was $0.73,0.88$ for uNGAL, 0.90 for MDA, 0.76 for leptin, and 0.91 for CysC. Adding new biomarkers to $\mathrm{SCr}$ enhanced the performance of DGF prediction, and the best combination was achieved with SCr, MDA, and CysC ( $A U C=0.96$, sensitivity $=100 \%$; specificity $=86 \%$ ).

Conclusion: A combination of graft damage biomarkers outperformed SCr in the early diagnosis of DGF, and the best performance was achieved by a triple-marker approach, using SCr, MDA, and CysC.

(c) 2015 The Canadian Society of Clinical Chemists. Published by Elsevier Inc. All rights reserved.

\section{Introduction}

Delayed graft function (DGF) is a clinical diagnosis that describes kidney dysfunction immediately after kidney transplantation (KTx), usually related to ischemic damage to the graft. The rate of DGF after KTx varies from $2 \%$ to $50 \%$, depending on the definition and the center's practice, and it is one of the most important risk factors for both acute rejection and impaired renal function at one year [1-3]. Ischemia/ reperfusion injury after organ transplantation is a major cause of DGF,

Abbreviations: AUC-ROC, area under the ROC curve; DGF, delayed graft function; CysC cystatin C; KTx, kidney transplantation; MDA, malondialdehyde; NGAL, neutrophi gelatinase-associated lipocalin; ROC, receiver-operating characteristic; $\mathrm{SCr}$, serum creatinine; uNGAL, urinary neutrophil gelatinase-associated lipocalin.

* Corresponding author at: Department of Nephrology and Kidney Transplantation, Centro Hospitalar do Porto, Hospital de Santo António, Largo Prof. Abel Salazar, 4099001 Porto, Portugal.

E-mail address: isabelf27@gmail.com (I. Fonseca). which is associated with prolonged hospital stay, additional invasive procedures, supplementary costs and greater risk of early and longterm graft loss [4]. Much research effort has been devoted to the search for DGF predictors, also called markers that would allow for early determination of allograft function and of prognoses for one-year and longterm graft survival. Unfortunately, the use of baseline characteristics alone (donor and recipient age, pre-transplant dialysis time, HLA mismatches, etc.) has limited accuracy in predicting both early and late graft outcomes. Several clinical algorithms have been proposed to predict DGF based on pre-operative risk factors, but none has been used routinely in clinical practice [5-7].

Currently, although inadequate for timely diagnosis and prognosis, serum creatinine ( $\mathrm{SCr}$ ) is an established and most commonly used clinical indicator of kidney function. Recent insights have suggested that allograft injury and recovery could be better characterized by new biomarkers. In KTx, numerous biomarkers have been evaluated over the past decade, but thus far, the evidence to support their use in routine 
practice has been limited [8-10]. Newly introduced biomarkers should complement and have incremental diagnostic and prognostic value over and above the current established markers. It would be ideal to discover a single marker with very high discriminatory accuracy, defined in terms of test sensitivity and specificity [11,12]. However, for most diseases, and particularly for KTx, single markers might not reflect all of the facets of initial graft dysfunction, and they do not have adequate sensitivity or specificity for practical purposes. One approach to increasing the clinical value of biomarkers and improving their screening sensitivity is to identify additional biomarkers and to combine them because a multimarker strategy might better characterize the complexity of DGF.

We recently demonstrated, in a prospective cohort study, that urinary neutrophil gelatinase-associated lipocalin (uNGAL) [13], serum cystatin C (CysC) and malondialdehyde (M.DA) [14], and serum leptin [15] within the first $24 \mathrm{~h}$ following KTx predicted DGF better than $\mathrm{SCr}$ levels or changes in $\mathrm{SCr}$ levels. Utilizing the same cohort, our primary goal for the current study was to investigate the value of combining these new markers with the widely used SCr level in predicting DGF (defined as dialysis requirement within the first week after KTx).

\section{Patients and methods}

\subsection{Study design and patient population}

Consecutive patients with end-stage renal disease, who were undergoing living or deceased donor KTx in the Department of Nephrology and Kidney Transplantation of Centro Hospitalar do Porto between December 2010 and May 2011, were prospectively enrolled. Patients younger than 18 years old or who required multiorgan transplants were not included. After transplantation, recipients with primary graft failure were excluded. The Institutional Review Board of Centro Hospitalar do Porto approved this study. Each participant provided informed consent.

\subsection{Sample collection and biomarker measurements}

Blood and urinary samples were collected as follows: 3 to $6 \mathrm{~h}$ prior to transplant surgery (pre-transplant); on the following morning, approximately 8 to $12 \mathrm{~h}$ after graft reperfusion (day-1); and then on the second, fourth, and seventh days after transplant, for a total of five samples per patient. All samples were immediately centrifuged, aliquoted and frozen within one hour after collection. Subsequently, they were stored at $-80{ }^{\circ} \mathrm{C}$ until analysis, which was performed approximately one to two weeks after collection.

The candidate biomarkers were measured using the following methods. Neutrophil gelatinase-associated lipocalin was measured in urine (uNGAL) using a two-step chemiluminescent microparticle immunoassay on a standardized clinical platform (ARCHITECT, Abbott Diagnostics, Germany). Malondialdehyde was measured in plasma using a commercial high-performance liquid chromatography kit (Chromsystems, Munich, Germany). Leptin was measured in serum by ELISA, based on the direct sandwich technique using kits from Mercodia (Sweden). Cystatin $C$ was measured in serum (CysC) by particle-enhanced nephelometric immunoassay (Siemens Diagnostics, Germany).

\subsection{Definitions}

Delayed graft function was defined by the need for dialysis during the first week after KTx. "Prompt" function (non-DGF) was characterized by no dialysis session being required during the first post-transplantation week.

Percentage change in $\mathrm{SCr}(\triangle \mathrm{SCr})$ was calculated individually for each subject as the difference between $\mathrm{SCr}$ on day- 1 and day- 0 divided by $\mathrm{SCr}$ on day- 0 (or difference between day- 2 and day- 1 divided by SCr on day1), multiplied by 100 .

\subsection{Statistical analyses}

The distributions of continuous variables were analyzed, and the Kolmogorov-Smirnov test was performed to assess their deviation from the normal distribution. Variables showing a positively skewed distribution, such as SCr, uNGAL, and serum leptin, were natural logarithm transformed [16] prior to parametric test analyses. Normally distributed variables are presented as the mean values and standard deviations (SDs), and variables exhibiting skewed distributions are presented as the medians and 25th-75th quartiles $(\mathrm{IQR}=$ interquartile range). Categorical variables are reported as percentages.

Correlations between biomarkers were assessed using Pearson's correlation coefficient, and $\mathrm{SCr}$, uNGAL, and serum leptin levels were logtransformed before analysis to normalize the distribution.

Receiver-operating characteristics (ROC) curves were generated to analyze single and multiple biomarkers, and the areas under the curve (AUCs) were calculated. For joint analysis of multiple biomarkers, a fitted multiple logistic regression model (with DGF as the dependent variable) was used to yield maximum sensitivity and specificity. Pretransplant variables known to be associated with DGF and considered potential confounders were included in the models. Multicollinearity among covariates was examined through the correlations of regression coefficients. The optimal cut-off points were determined by the largest sum of sensitivity and specificity, and the positive and negative likelihood ratios were calculated as follows: sensitivity/(1-specificity) and (1-sensitivity)/specificity, respectively [17].

Statistical analyses were performed using the SPSS software, version 22.0 , and a significance level of 0.05 was considered.

\section{Results}

During the recruitment period, 42 patients were enrolled consecutively. Two recipients had primary graft failure and were excluded. Therefore, the final study cohort included 40 patients. Their demographic and transplant data are shown in Table 1.

Eighteen (45\%) patients had DGF, whereas 22 (55\%) patients experienced prompt graft function. In terms of the traditional predictors of DGF, except for cold ischemia time and recipient age, no significant differences were found between the DGF and non-DGF groups regarding the baseline characteristics or induction therapy (Table 1). The rate of DGF was higher in kidney grafts from deceased donors, but this difference was not statistically significant $(51.7 \%$ vs. $27.3 \%, P=0.286)$. The median levels of SCr at day-7 after KTx were higher in recipients with grafts from deceased donors compared to living donors, but this difference was not statistically significant (2.96 vs. $1.95 \mathrm{mg} / \mathrm{dL}$, $P=0.303)$.

\subsection{Candidate biomarkers for the detection of DGF}

Detailed results regarding each biomarker were previously reported [13-15]. Briefly, levels of uNGAL, serum MDA, leptin and CysC were significantly higher in DGF patients. Longitudinal changes in the four biomarkers, according to graft function within the first week after KTx, are shown in Fig. 1. The correlations between markers are displayed in Table 2. All of the biomarkers were positively correlated, with the exception of leptin.

3.2. ROC analysis of day-1 SCr, uNGAL, and serum CysC, MDA, and leptin, individually and combined as early biomarkers for DGF detection

Receiver operating characteristic curves were generated, and AUCs were calculated to estimate the diagnostic accuracy of each marker for DGF. Based on the logistic models, the predicted values of the combined predictors were calculated and analyzed using ROC curves. The performance of day-1 $\mathrm{SCr}$ alone and in association with each of the new 
Table 1

Summary of the demographic and clinical characteristics of the kidney transplant donors and recipients (total patient cohort and separated into delayed or prompt graft function)

\begin{tabular}{|c|c|c|c|c|}
\hline & $\begin{array}{l}\text { Total } \\
(\mathrm{n}=40)\end{array}$ & $\begin{array}{l}\text { DGF } \\
(\mathrm{n}=18)\end{array}$ & $\begin{array}{l}\text { Non-DGF } \\
(\mathrm{n}=22)\end{array}$ & $P$-value \\
\hline \multicolumn{5}{|l|}{ Donor } \\
\hline Age (year) & $51.2 \pm 11.4$ & $51.1 \pm 13.4$ & $51.2 \pm 9.9$ & 0.172 \\
\hline Male sex & $26(65)$ & $14(78)$ & $12(54.5)$ & 0.125 \\
\hline Living donor & $11(27.5)$ & $3(16.7)$ & $8(36.4)$ & 0.165 \\
\hline Expanded criteria donors & $3(7.5)$ & $1(5.6)$ & $2(9.1)$ & 0.541 \\
\hline Serum creatinine $(\mathrm{mg} / \mathrm{dL})$ & $0.81 \pm 0.18$ & $0.85 \pm 0.21$ & $0.78 \pm 0.16$ & 0.318 \\
\hline \multicolumn{5}{|l|}{ Donor-recipient } \\
\hline HLA mismatches & $3.39 \pm 1.24$ & $3.38 \pm 1.07$ & $3.41 \pm 1.46$ & 0.941 \\
\hline Cold ischemia time $(\mathrm{h})$ & $12.1 \pm 7.9$ & $15.2 \pm 7.8$ & $9.6 \pm 7.3$ & $0.035^{*}$ \\
\hline Living donor & $2.8 \pm 0.5$ & $2.5 \pm 0.5$ & $3.0 \pm 0.5$ & 0.204 \\
\hline Deceased donor & $16.2 \pm 5.9$ & $18.1 \pm 5.1$ & $14.1 \pm 6.2$ & 0.088 \\
\hline \multicolumn{5}{|l|}{ Recipient } \\
\hline Age (year) & $49.2 \pm 15.2$ & $56.3 \pm 10.9$ & $43.3 \pm 15.9$ & $0.006^{*}$ \\
\hline Male sex & $26(65)$ & $11(61)$ & $15(68)$ & 0.641 \\
\hline Caucasian & $40(100)$ & $18(100)$ & $22(100)$ & - \\
\hline BMI $\left(\mathrm{kg} / \mathrm{m}^{2}\right)$ & $24.8 \pm 4.9$ & $26.2 \pm 4.4$ & $23.6 \pm 5.0$ & 0.091 \\
\hline Previous kidney transplant & $2(5)$ & $0(0)$ & $2(9.1)$ & \\
\hline Time on dialysis (year) & $4.4 \pm 4.7$ & $5.6 \pm 6.2$ & $3.4 \pm 2.3$ & 0.135 \\
\hline \multicolumn{5}{|l|}{ Pre-transplant therapy } \\
\hline Dialysis & $38(95)$ & $18(100)$ & $20(90.9)$ & 0.296 \\
\hline Pre-emptive transplantation & $2(5)$ & $0(0)$ & $2(9.1)$ & \\
\hline \multicolumn{5}{|l|}{ Cause of kidney disease } \\
\hline IgA nephropathy & $7(17.5)$ & $2(11.1)$ & $5(22.7)$ & - \\
\hline Glomerulonephritis & $6(15.0)$ & $4(22.2)$ & $2(9.1)$ & - \\
\hline Diabetic nephropathy & $5(12.5)$ & $3(16.7)$ & $2(9.1)$ & - \\
\hline Autosomal-dominant polycystic kidney disease & $3(7.5)$ & $3(16.7)$ & $0(0)$ & - \\
\hline Unknown & $4(10.0)$ & $1(5.6)$ & $3(13.6)$ & - \\
\hline Others & $15(37.5)$ & $5(27.8)$ & $10(45.5)$ & - \\
\hline Peak PRA (\%) & $5.5 \pm 15.1$ & $5.0 \pm 15.0$ & $5.9 \pm 15.5$ & 0.853 \\
\hline 0 & $29(72.5)$ & $14(77.8)$ & $15(68.2)$ & - \\
\hline $1-25$ & $8(20.0)$ & $3(16.7)$ & $5(22.7)$ & - \\
\hline $26-75$ & $3(7.5)$ & $1(5.6)$ & $2(9.0)$ & - \\
\hline Current PRA (\%) & $2.3 \pm 8.6$ & $3.1 \pm 11.7$ & $1.6 \pm 4.9$ & 0.585 \\
\hline 0 & $34(85)$ & $15(83.3)$ & $19(86.4)$ & - \\
\hline $1-25$ & $5(12.5)$ & $2(11.1)$ & $3(13.6)$ & - \\
\hline $26-50$ & $1(2.5)$ & $1(5.6)$ & $0(0)$ & - \\
\hline \multicolumn{5}{|l|}{ Induction regimen } \\
\hline Antithymocyte globulin (ATG-F) & $4(10)$ & $1(5.6)$ & $3(13.6)$ & 0.613 \\
\hline Basiliximab/daclizumab & $30(75)$ & $14(77.8)$ & $16(72.7)$ & 0.789 \\
\hline \multicolumn{5}{|l|}{ Immunosuppression at the time of discharge } \\
\hline Steroids & $38(95.0)$ & $18(100)$ & $20(90.9)$ & 0.296 \\
\hline Tacrolimus & $38(95.0)$ & $17(94.4)$ & $21(95.5)$ & 0.886 \\
\hline Cyclosporine A & $2(0.05)$ & $1(5.6)$ & $1(5.6)$ & 0.884 \\
\hline
\end{tabular}

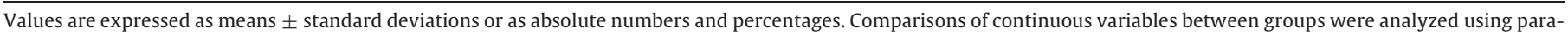
metric (t-test) or nonparametric (Mann-Whitney) tests; the associations between categorical variables were analyzed using the $\chi^{2}$ test and Fisher's exact test.

Abbreviations: HLA, human leukocyte antigen; BMI, body mass index; PRA, panel reactive antibody.

* $\mathrm{P}<0.05$

markers (uNGAL, serum CysC, MDA, and leptin) in diagnosing DGF is illustrated in Table 3. On the morning following KTx, the AUCs for the diagnosis of DGF using only $\mathrm{SCr}$ or $\triangle \mathrm{SCr}$ were 0.732 and 0.755 . When adding each of the new markers to $\mathrm{SCr}$, the performance of the combined markers improved substantially over that of the SCr individually, and the AUCs were 0.914 for CysC, 0.902 for MDA, 0.878 for UNGAL, and 0.792 for leptin. Combining SCr with the four new markers, the AUC was 0.95 , and the best diagnostic performance was for $\mathrm{SCr}$ combined with serum CysC and MDA (AUC $=0.96$ ).

After including $\mathrm{SCr}$, CysC, and MDA in a logistic model for combining these markers, none of the pre-transplant variables traditionally associated with DGF, namely donor and recipient age, pre-transplant dialysis time, HLA mismatches, and cold ischemia time, were statistically significant, and they were removed from the final model by backward elimination (Table 4). Recipient and donor age were included in the models separately, due to multicollinearity observed when both variables were included. For the same reason, given the multicollinearity when pre-transplant time on dialysis and cold ischemia time were entered into the model, time on dialysis was included, and donor status was used instead of cold ischemia time.
3.3. Performance of the triple-biomarker approach, including SCr, MDA, and CysC

For DGF ( $\mathrm{n}=18$ ) versus all non-DGF $(\mathrm{n}=22$ ), the formula achieved by fitting the multiple logistic regression model for combining $\mathrm{SCr}$, MDA, and CysC measured 8 to $12 \mathrm{~h}$ after KTx was as follows: $(-12.062+0.152 \times \mathrm{SCr}+1.429 \times \mathrm{CysC}+16.789 \times \mathrm{MDA})$.

Using this formula and calculating the predicted values, the optimal sensitivity and specificity occurred at a value of 0.278 (sensitivity: $100 \%$; specificity: $86 \%$; positive and negative predictive value: $88 \%$ and $100 \%$, respectively; and positive and negative likelihood ratios of 7.35 and 0 , respectively).

\section{Discussion}

We investigated the usefulness of combining $\mathrm{SCr}$ with four new biomarkers for predicting DGF after KTx. A triple-marker approach, using $\mathrm{SCr}$, CysC, and MDA measured 8 to $12 \mathrm{~h}$ after KTx, was the most informative combination, resulting in an increased ability $($ AUC $=0.96)$ to 

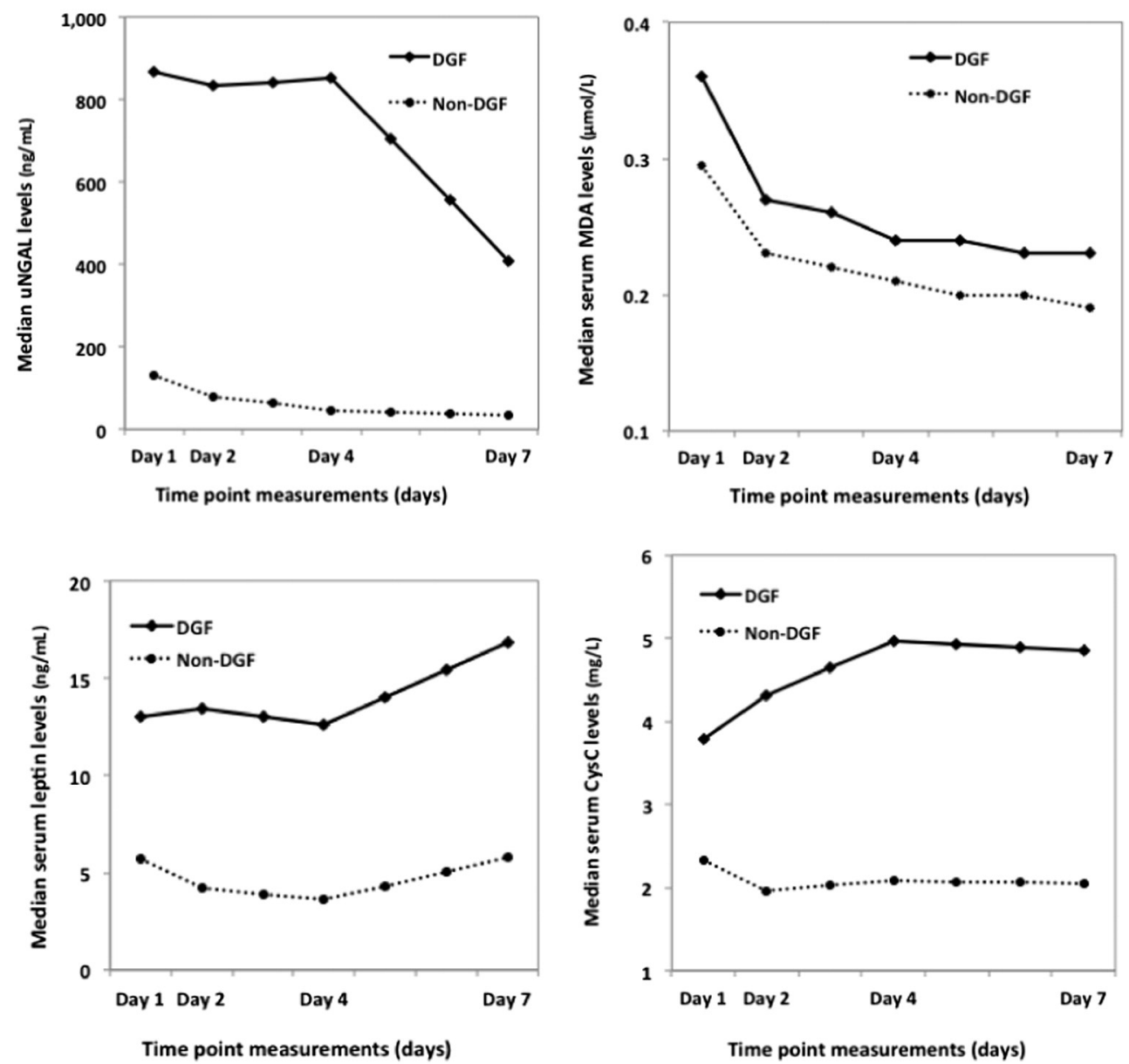

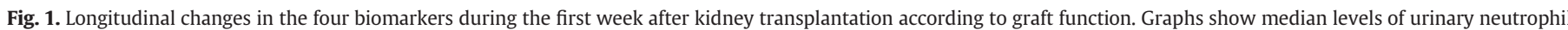

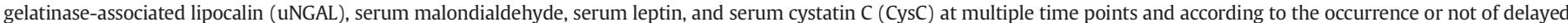
graft function (DGF and non-DGF, respectively).

distinguish patients with graft damage and those who would require dialysis within the first week.

Allograft function following KTx is commonly monitored using $\mathrm{SCr}$, even given the inaccuracy of this marker due to the impact of age, sex, and muscle mass on creatinine generation and its inability to detect functional impairment of less than 50\% [18]. These limitations hamper $\mathrm{SCr}$ from being a precise index of renal allograft function and compromise its value as a sensitive and reliable tool in the clinical management of KTx recipients. Therefore, interest has arisen in alternative markers of renal function, and CysC is an emerging endogenous marker of glomerular filtration rate. Cystatin $C$ is not affected by other physiological or pathological changes and is then freely filtered by the glomeruli without tubular reabsorption or secretion. Cystatin $C$ meets all of the criteria of a reliable marker of glomerular filtration rate, and several studies have described the superiority of serum CysC over SCr for diagnosing filtration failure, both in native kidney disease patients $[19,20]$ and in KTx recipients [21-23].

Although not a marker of glomerular filtration, NGAL, in both the urine and plasma, has been considered a promising biomarker of AKI and DGF in several settings [24,25]. NGAL is normally expressed at low levels in multiple tissues, and it is excreted in the urine in small amounts. Nevertheless, after renal insult, both urine and plasma NGAL are increased, as occurs following ischemia-reperfusion injury in transplanted kidneys. Induction of NGAL after kidney injury precedes the elevation of classical markers of kidney damage. Given these data, levels of NGAL were shown to predict dialysis requirements within the first week, notably preceding the postoperative peak in $\mathrm{SCr}$ levels, which only occurred between the second and fourth days [8,13,26,27].

Table 2

Correlation between markers measured on the first day after kidney transplantation.

\begin{tabular}{|c|c|c|c|c|c|c|c|}
\hline & $\mathrm{SCr}(\ln )$ & $\Delta \mathrm{SCr}_{\text {day1-day0 }}$ & $\Delta \mathrm{SCr}_{\text {day2-day1 }}$ & uNGAL (ln) & MDA & Leptin $(\ln )$ & CysC \\
\hline $\mathrm{SCr}(\ln )$ & - & 0.17 & 0.07 & $0.55^{*}$ & $0.45^{*}$ & -0.10 & $0.51^{*}$ \\
\hline$\Delta \mathrm{SCr}_{\text {day1-day0 }}$ & 0.17 & - & $0.42^{*}$ & 0.49 & $0.46^{*}$ & 0.02 & $0.60^{* *}$ \\
\hline$\Delta \mathrm{SCr}_{\text {day2-day1 }}$ & 0.07 & $0.42^{*}$ & - & $0.63^{* *}$ & $0.32^{\dagger}$ & 0.16 & $0.45^{*}$ \\
\hline uNGAL (ln) & $0.55^{*}$ & $0.49^{*}$ & $0.63^{* *}$ & - & $0.52^{*}$ & 0.25 & $0.80^{* *}$ \\
\hline MDA & $0.45^{*}$ & $0.46^{*}$ & $0.32^{\dagger}$ & $0.52^{*}$ & - & 0.12 & $0.58^{* *}$ \\
\hline Leptin $(\ln )$ & -0.10 & 0.02 & 0.16 & 0.25 & 0.12 & - & 0.15 \\
\hline CysC & $0.51^{*}$ & $0.60^{* *}$ & $0.45^{*}$ & $0.80^{* *}$ & $0.58^{* *}$ & 0.15 & - \\
\hline
\end{tabular}

Values are given as Pearson's coefficients and relevant $P$-values. ${ }^{\dagger} P<0.05 ;{ }^{*} P<0.01 ;{ }^{* *} P<0.001$. Logarithm-transformed values of SCr, uNGAL, and leptin were used for analysis. Abbreviations: $\mathrm{SCr}=$ serum creatinine; uNGAL = urinary neutrophil gelatinase-associated lipocalin; MDA = serum malondialdehyde; CysC = serum cystatin C; In = logarithm. $\Delta \mathrm{SCr}_{\text {day1-day0 }}$ and $\Delta \mathrm{SCr}_{\text {day2-day1 }}=$ percentage changes in $\mathrm{SCr}$ between day-1 and day-0 and between day-2 and day-1. 
Table 3

Areas under the curve (AUCs) for single and combined biomarkers measured on the first day after kidney transplantation.

\begin{tabular}{llr}
\hline & AUC $(95 \% \mathrm{CI})$ & $P$-value \\
\hline Day 1 ( 8 to 12 h after KTx surgery) & & \\
Single & & \\
SCr $(\mathrm{mg} / \mathrm{dL})$ & $0.732(0.577-0.888)$ & 0.012 \\
$\Delta \mathrm{SCr}_{\text {day1-day0 }}(\%)$ & $0.755(0.603-0.907)$ & 0.006 \\
$\Delta \mathrm{SCr}$ day2-day1 $\%)$ & $0.783(0.643-0.923)$ & 0.002 \\
uNGAL $(\mathrm{ng} / \mathrm{mL})$ & $0.884(0.773-0.996)$ & $<0.001$ \\
MDA $(\mu \mathrm{mol} / \mathrm{L})$ & $0.900(0.807-0.994)$ & $<0.001$ \\
Leptin $(\mathrm{ng} / \mathrm{mL})$ & $0.759(0.597-0.921)$ & 0.008 \\
CysC $(\mathrm{mg} / \mathrm{L})$ & $0.914(0.822-1.000)$ & $<0.001$ \\
Combined & & \\
SCr + uNGAL & $0.878(0.763-0.992)$ & $<0.001$ \\
SCr + MDA & $0.902(0.811-0.992)$ & $<0.001$ \\
SCr + leptin & $0.792(0.649-0.935)$ & 0.003 \\
SCr + CysC & $0.914(0.820-1.000)$ & $<0.001$ \\
SCr + CysC + MDA & $0.960(0.905-1.000)$ & $<0.001$ \\
SCr + CysC + uNGAL & $0.918(0.823-1.000)$ & $<0.001$ \\
SCr + CysC + MDA + uNGAL & $0.939(0.863-1.000)$ & $<0.001$ \\
SCr + CysC + MDA + uNGAL + leptin & $0.950(0.882-1.000)$ & $<0.001$ \\
\hline
\end{tabular}

Abbreviations: $\mathrm{SCr}=$ serum creatinine; $\mathrm{uNGAL}=$ urinary neutrophil gelatinase-associated lipocalin; $\mathrm{MDA}=$ serum malondialdehyde; CysC $=$ serum cystatin $\mathrm{C} ; \mathrm{ln}=$ logarithm. $\Delta \mathrm{SCr}_{\text {day1-day0 }}$ and $\Delta \mathrm{SCr}_{\text {day2-day1 }}=$ percentage changes in $\mathrm{SCr}$ between day 1 and day 0 and between day 2 and day 1 .

Malondialdehyde was recently identified by our group as an early marker of graft dysfunction and a predictor of DGF [14]. Malondialdehyde is the most studied product of polyunsaturated fatty acid peroxidation and one of the best markers to detect oxidative stress [28]. Oxidative stress is one of the most important components of the ischemiareperfusion process [29-31], which is an inevitable phenomenon in KTx. Accordingly, we showed that elevated MDA levels reflected dysfunction of the kidney and predicted the need for renal replacement therapy within the first week following KTx better than SCr [14].

Along with MDA, leptin was another biomarker that was first hypothesized by our group to be an indicator of graft dysfunction. So far, no reported studies have examined the clinical utility of leptin in the diagnosis of graft dysfunction after KTx. To the best of our knowledge, we addressed this question for the first time, and we confirmed that leptin levels slightly outperformed the traditional biomarker $\mathrm{SCr}$ in assessing the prognosis of DGF [15].

Our previous research concentrated on single biomarkers for DGF diagnosis. The present study focused on combining biomarkers to diagnose DGF more efficiently. Due to the complexity and overlapping pathophysiological mechanisms contributing to DGF and the distinct expression of biomarkers, it is unlikely that a single biomarker would be sufficient for accurate and reliable diagnosis of graft damage. Consequently, the incorporation of several markers into a diagnostic or prognostic panel will likely be required to profile acute graft injury and its effects [32,33]. Malondialdehyde and CysC evaluate different aspects and/or areas of kidney injury: MDA appears to be a more specific marker of ischemic-reperfusion, whereas CysC is a more functional marker of the glomerular filtration rate rather than of injury. Given these findings,

Table 4

Results of multivariable logistic regression analysis of predictors of delayed graft function, after backward elimination of variables routinely associated with delayed graft function.

\begin{tabular}{llll}
\hline & OR & $P$-value & $95 \% \mathrm{CI}$ \\
\hline SCr (per unit increase) & 1.164 & 0.572 & $0.688-1.970$ \\
Cystatin C (per unit increase) & 4.176 & 0.023 & $1.219-14.303$ \\
MDA (per 10 unit increase) & 5.360 & 0.048 & $1.016-28.276$
\end{tabular}

Note: Results given by logistic regression. Recipient age, pre-transplant time on dialysis, HLA mismatches, and donor status (living/deceased) were not retained in the final model after backward elimination. Serum creatinine was forced to be included in the model, using the enter method.

Abbreviations: OR = odds ratio; 95\% CI (95\% confidence interval); $\mathrm{SCr}=$ serum creatinine; $\mathrm{MDA}=$ serum malondialdehyde; CysC $=$ serum cystatin $\mathrm{C}$. combining these two biological pathways through these markers seems to increase their predictive value beyond that of $\mathrm{SCr}$ in providing an early diagnosis of DGF.

Although uNGAL has shown promising results as a predictor of DGF, the performance of the combinations that comprise UNGAL was lower than the triple-marker approach with SCr, MDA, and CysC. A possible explanation for the inferior performance of UNGAL might have been the lack of samples in some recipients with transient anuria. In our study, 5 recipients were anuric the morning following graft reperfusion, resulting in $12.5 \%$ of our patients not having urine samples to measure uNGAL at that particular time point. These patients could not be included in any of the approaches that included uNGAL, which reduced the sample size and the performance of UNGAL combinations. We chose to measure NGAL in urine instead of blood because UNGAL more accurately represents tubule damage in the kidney than filtration from the blood and because of the non-invasive nature of sample collection and the reduced number of interfering proteins. However, despite the unquestionable value of urinary markers, their use in transplant recipients could also be a drawback because of possible transient graft anuria.

This paper explored the optimal marker combination for DGF diagnosis using logistic regression. Combining multiple biomarkers for clinical use remains a challenge. The additional diagnostic and prognostic information gained by any biomarker over an established marker must be determined using adequate statistical tools. We focused particularly on improving the sensitivity and specificity in order to increase the accuracy of the diagnosis of acute graft dysfunction and of dialysis requirement prognosis. A very large number of biomarkers fail to be used in clinical practice due to the weakness of their clinical performance, namely, low sensitivity and low specificity [34]. The current study identified a simple and clinically applicable tool for detecting graft dysfunction earlier than $\mathrm{SCr}$ alone. For practical purposes, the concentrations of SCr, MDA, and CysC can be easily measured in routine blood samples, and the results can be replaced in the fitted formula. The final score would allow for the identification of kidneys with significant ischemia-reperfusion injury, which could result in easier clinical decision-making and more effective recipient management, thereby improving outcomes.

We previously described the strengths and limitations of this observational study. Given our relatively small sample size, larger and multicenter studies should be conducted in the transplant setting to verify our findings. Despite limitations, the current results suggested that the combined use of SCr, MDA, and CysC could be an important tool for the early determination of allograft function and thereafter for identifying individuals at the greatest risk of developing DGF. A major problem in selecting a biomarker profile is the proportional increase in economic burden. Thus, a "parsimonious" biomarker combination must be used in a cost-effective manner, and this consideration was an additional reason for choosing a combination of two, and not more, new markers to be added to the traditional marker.

In summary, combining biomarkers from different pathophysiologic pathways seems to be rational and a reliable strategy for optimizing sensitivity and specificity and obtaining additive diagnostic and prognostic information. In this single-center study using a relatively small cohort of patients, we used multiple logistic regressions to combine several biomarkers, and a triple-marker approach, using SCr, MDA, and CysC, showed added value for the early detection of DGF.

\section{Funding sources}

The authors recognize and thank Astellas Pharma Portugal for its valuable contributions in supporting the acquisition of reagents for measuring MDA and Abbott Laboratories for providing the kits for measuring urinary NGAL in almost 200 samples. The remaining NGAL kits were financed by the Multidisciplinary Unit for Biomedical Investigation, Porto, Portugal, which was funded by grants from the Foundation for Science and Technology (Fcomp-01-0124-FEDER-015896). 


\section{Disclosure policy}

The authors declare no conflicts of interest regarding the publication of this article.

The results presented in this paper have not been published previously, in whole or in part.

Role of the Sponsors: Astellas Pharma Portugal and Abbot Laboratories played no roles in the design or conducting of the study, the collection, management, analysis, or interpretation of the data, or the preparation or approval of the manuscript.

\section{Author contributions}

Isabel Fonseca had full access to the data from the study and takes responsibility for the integrity of the data and the accuracy of the data analysis.

Study concept and design: Isabel Fonseca.

Acquisition of the data and patient recruitment: Isabel Fonseca, Manuela Almeida, La Salete Martins, Leonídio Dias, Josefina Santos, Sofia Pedroso, and António Castro Henriques.

Technical support and laboratory analysis: Henrique Reguengo and José Carlos Oliveira.

Statistical analysis and interpretation of the data: Isabel Fonseca and Denisa Mendonça.

Drafting of the manuscript: Isabel Fonseca.

Critical revision of the manuscript for important intellectual content: Denisa Mendonça, Jorge Malheiro, and António Castro Henriques; all of the authors approved the final version.

Study supervision: Denisa Mendonça, António Castro Henriques, and Luísa Lobato.

\section{Acknowledgments}

The investigators thank Dr. António Cabrita for permitting the study and the nursing and laboratory staffs for their invaluable contributions to this work.

\section{References}

[1] L.E. Kyllonen, K.T. Salmela, B.H. Eklund, L.E. Halme, K.A. Hockerstedt, H.M. Isoniemi, et al., Long-term results of 1047 cadaveric kidney transplantations with special emphasis on initial graft function and rejection, Transpl. Int. 13 (2000) 122-128.

[2] A.O. Ojo, J.A. Hanson, R.A. Wolfe, A.B. Leichtman, L.Y. Agodoa, F.K. Port, Long-term survival in renal transplant recipients with graft function, Kidney Int. 57 (2000) 307-313.

[3] A.O. Ojo, R.A. Wolfe, P.J. Held, F.K. Port, R.L. Schmouder, Delayed graft function: risk factors and implications for renal allograft survival, Transplantation 63 (1997) 968-974.

[4] C. Ponticelli, Ischaemia-reperfusion injury: a major protagonist in kidney transplantation, Nephrol. Dial. Transplant. 29 (2014) 1134-1140.

[5] E. Rodrigo, E. Minambres, J.C. Ruiz, A. Ballesteros, C. Pinera, J. Quintanar, et al., Prediction of delayed graft function by means of a novel web-based calculator: a single-center experience, Am. J. Transplant. 12 (2012) 240-244.

[6] W.D. Irish, D.A. McCollum, R.J. Tesi, A.B. Owen, D.C. Brennan, J.E. Bailly, et al., Nomogram for predicting the likelihood of delayed graft function in adult cadaveric renal transplant recipients, J. Am. Soc. Nephrol. 14 (2003) 2967-2974.

[7] G. Machnicki, B. Pinsky, S. Takemoto, R. Balshaw, P.R. Salvalaggio, P.M. Buchanan, et al., Predictive ability of pretransplant comorbidities to predict long-term graft loss and death, Am. J. Transplant. 9 (2009) 494-505.

[8] C.R. Parikh, A. Jani, J. Mishra, Q. Ma, C. Kelly, J. Barasch, et al., Urine NGAL and IL-18 are predictive biomarkers for delayed graft function following kidney transplantation, Am. J. Transplant. 6 (2006) 1639-1645.

[9] M.P. Welberry Smith, A. Zougman, D.A. Cairns, M. Wilson, T. Wind, S.L. Wood, et al Serum aminoacylase- 1 is a novel biomarker with potential prognostic utility for long-term outcome in patients with delayed graft function following renal transplantation, Kidney Int. 84 (2013) 1214-1225.

[10] G. Zaza, F. Rascio, P. Pontrelli, S. Granata, P. Stifanelli, M. Accetturo, et al., Karyopherins: potential biological elements involved in the delayed graft function in renal transplant recipients, BMC Med. Genomics 7 (2014) 14.

[11] N.R. Cook, Statistical evaluation of prognostic versus diagnostic models: beyond the ROC curve, Clin. Chem. 54 (2008) 17-23.

[12] J.L. Gastwirth, The statistical precision of medical testing procedures: applications to polygraph/AIDS antibodies test data, Stat. Sci. 2 (1987) 213-238.

[13] I. Fonseca, J.C. Oliveira, M. Almeida, M. Cruz, A. Malho, L.S. Martins, et al., Neutrophil gelatinase-associated lipocalin in kidney transplantation is an early marker of graft dysfunction and is associated with one-year renal function, J. Transplant. 2013 (2013) 650123.

[14] I. Fonseca, H. Reguengo, M. Almeida, L. Dias, L.S. Martins, S. Pedroso, et al., Oxidative stress in kidney transplantation: malondialdehyde is an early predictive marker of graft dysfunction, Transplantation 97 (2014) 1058-1065.

[15] I. Fonseca, J.C. Oliveira, J. Santos, J. Malheiro, L.S. Martins, M. Almeida, et al., Leptin and adiponectin during the first week after kidney transplantation: biomarkers of graft dysfunction? Metabolism 64 (2015) 202-207.

[16] T.P. Carter, K.C. Spitalny, N.R. Marsh, N. Eaton, S.A. Herrick, M.H. Riser, Comprehensive statewide approach to improve youth outcomes: experience of the New York State Youth Development Team, J. Public Health Manag. Pract. (2006) S32-S40 (Suppl.).

[17] D.A. Grimes, K.F. Schulz, Refining clinical diagnosis with likelihood ratios, Lancet 365 (2005) 1500-1505.

[18] J.L. Slocum, M. Heung, S. Pennathur, Marking renal injury: can we move beyond serum creatinine? Transl. Res. 159 (2012) 277-289.

[19] V.R. Dharnidharka, C. Kwon, G. Stevens, Serum cystatin C is superior to serum creatinine as a marker of kidney function: a meta-analysis, Am. J. Kidney Dis. 40 (2002) 221-226.

[20] J.F. Roos, J. Doust, S.E. Tett, C.M. Kirkpatrick, Diagnostic accuracy of cystatin C compared to serum creatinine for the estimation of renal dysfunction in adults and children-a meta-analysis, Clin. Biochem. 40 (2007) 383-391.

[21] U. Poge, T. Gerhardt, B. Stoffel-Wagner, H. Palmedo, H.U. Klehr, T. Sauerbruch, et al., Cystatin C-based calculation of glomerular filtration rate in kidney transplant recipients, Kidney Int. 70 (2006) 204-210.

[22] C. White, A. Akbari, N. Hussain, L. Dinh, G. Filler, N. Lepage, et al., Estimating glomerular filtration rate in kidney transplantation: a comparison between serum creatinine and cystatin C-based methods, J. Am. Soc. Nephrol. 16 (2005) 3763-3770.

[23] N. Maillard, C. Mariat, C. Bonneau, M. Mehdi, L. Thibaudin, S. Laporte, et al., Cystatin C-based equations in renal transplantation: moving toward a better glomerular filtration rate prediction? Transplantation 85 (2008) 1855-1858.

[24] M. Haase, R. Bellomo, P. Devarajan, P. Schlattmann, A. Haase-Fielitz, Accuracy of neutrophil gelatinase-associated lipocalin (NGAL) in diagnosis and prognosis in acute kidney injury: a systematic review and meta-analysis, Am. J. Kidney Dis. 54 (2009) 1012-1024.

[25] A. Buemi, F. Musuamba, S. Frederic, A. Douhet, M. De Meyer, L. De Pauw, et al., Is plasma and urine neutrophil gelatinase-associated lipocalin (NGAL) determination in donors and recipients predictive of renal function after kidney transplantation? Clin. Biochem. 47 (2014) 68-72.

[26] M.E. Hollmen, L.E. Kyllonen, K.A. Inkinen, M.L. Lalla, K.T. Salmela, Urine neutrophil gelatinase-associated lipocalin is a marker of graft recovery after kidney transplantation, Kidney Int. 79 (2011) 89-98.

[27] D. Bolignano, V. Donato, G. Coppolino, S. Campo, A. Buemi, A. Lacquaniti, et al., Neutrophil gelatinase-associated lipocalin (NGAL) as a marker of kidney damage, Am. J. Kidney Dis. 52 (2008) 595-605.

[28] D. Del Rio, A.J. Stewart, N. Pellegrini, A review of recent studies on malondialdehyde as toxic molecule and biological marker of oxidative stress, Nutr. Metab. Cardiovasc. Dis. 15 (2005) 316-328.

[29] D.K. de Vries, K.A. Kortekaas, D. Tsikas, L.G. Wijermars, C.J. van Noorden, M.T. Suchy, et al., Oxidative damage in clinical ischemia/reperfusion injury: a reappraisal, Antioxid. Redox Signal. 19 (2013) 535-545.

[30] J. Kim, Y.M. Seok, K.J. Jung, K.M. Park, Reactive oxygen species/oxidative stress contributes to progression of kidney fibrosis following transient ischemic injury in mice, Am. J. Physiol. Renal. Physiol. 297 (2009) F461-F470.

[31] H.L. Waller, S.J. Harper, S.A. Hosgood, A. Bagul, B. Yang, M.D. Kay, et al., Biomarkers of oxidative damage to predict ischaemia-reperfusion injury in an isolated organ perfusion model of the transplanted kidney, Free Radic. Res. 40 (2006) 1218-1225.

[32] A. Siedlecki, W. Irish, D.C. Brennan, Delayed graft function in the kidney transplant, Am. J. Transplant. 11 (2011) 2279-2296.

[33] K. Maiese, Marking the onset of oxidative stress: biomarkers and novel strategies, Oxidative Med. Cell. Longev. 2 (2009) 1.

[34] N.G. Frangogiannis, Biomarkers: hopes and challenges in the path from discovery to clinical practice, Transl. Res. 159 (2012) 197-204. 